\title{
A nonlinearities inverse distance weighting spatial interpolation approach applied to the surface electromyography signal
}

\author{
Ayad Assad Ibrahim, Ikhlas Mahmoud Farhan, Mohammed Ehasn Safi \\ Department of Electrical Engineering, Electronics Engineering Branch, University of Technology-Iraq, Baghdad, Iraq
}

\section{Article Info}

Article history:

Received Mar 22, 2021

Revised Sep 20, 2021

Accepted Oct 7, 2021

\section{Keywords:}

Electromyography nonlinearities-interpolation spatial interpolation

Goodness of fit

Inverse distance weighted

ABSTRACT

Spatial interpolation of a surface electromyography (sEMG) signal from a set of signals recorded from a multi-electrode array is a challenge in biomedical signal processing. Consequently, it could be useful to increase the electrodes' density in detecting the skeletal muscles' motor units under detection's vacancy. This paper used two types of spatial interpolation methods for estimation: Inverse distance weighted (IDW) and Kriging. Furthermore, a new technique is proposed using a modified nonlinearity formula based on IDW. A set of EMG signals recorded from the noninvasive multi-electrode grid from different types of subjects, sex, age, and type of muscles have been studied when muscles are under regular tension activity. A goodness of fit measure (R2) is used to evaluate the proposed technique. The interpolated signals are compared with the actual signals; the Goodness of fit measure's value is almost $99 \%$, with a processing time of $100 \mathrm{msec}$. The resulting technique is shown to be of high accuracy and matching of spatial interpolated signals to actual signals compared with IDW and Kriging techniques.
\end{abstract}

This is an open access article under the CC BY-SA license.

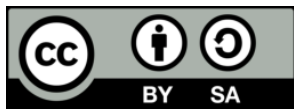

\section{Corresponding Author:}

Ayad Assad Ibrahim

Department of Electrical Engineering, University of Technology-Iraq

Baghdad, Iraq

Email: 30055@uotechnology.edu.iq

\section{INTRODUCTION}

The surface electromyography (EMG) signal is the spatial and temporal summation of all active motor units' action potential under regional detection's vacancy [1], [2]. A high-spatial-precision EMG electrode, mounted on the skin surface of skeletal muscle, allows detection and estimation of the size of a single motor unit (MU). Moreover, analyzes neurological properties like conduct velocity single of the motor unit action potential (MUAP) activity when a muscular tissue is under contraction [3]. This paper's main approach to enhance the surface EMG signal is based on spatial interpolation. The simple interpolation approach is to find the closest data value and to give the same value. This approach is not likely to be used as a linear interpolation [4]. Estimating missing data is an essential issue in signal processing [5]. Spatial interpolation has been commonly and frequently utilized in many analyses to obtain surface data dependent on a set of sampled points, like temperature, soil properties, and precipitation [6]. Consequently, spatial interpolation is one of the engineering tools to estimate spatial missing information in many signals like EMG. There are many researchers in both of those hot fields, enhancement of spatial detectors, and optimization of Spatial Interpolation, in the last few years.

Abser et al. [7] illustrate a comparison between two interpolation methods, raw spectrum interpolation, and smooth spectrum interpolation, for error estimation signal-to- $60 \mathrm{~Hz}$-noise ratio surface EMG signals. The results show that the smoothed spectrum is relatively better than the raw spectrum, but 
both methods have estimation errors $<10 \%$ relative to signal power. $\mathrm{Hu}$ et al. [8] proposes an interpolation method (RicFlow) of correspondence for noise cancellation. RicFlow has the smallest expected prediction error (EPE) relative to the other methods. The proposed method has (5.620) EPE. However, it still failed when low-density matches as input are applied. Martinez et al. [9] proposes a method to estimate grasp force (GF) using 16 EMG channels. Two Reduction methods, the Fix-EN and LassoG-EN, are used to select the number of features, to achieve between $90 \%$ and $99 \%$ of the initial number of features. According to the training windows (TW) length, the prediction improved until a stable performance was reached around 400 ms after the grasp force (GF) onset. Li et al. [10] proposes an optimized method of inverse distance weighted spatial interpolation (IDW) called an adjusted inverse distance weighted spatial interpolation method (AIDW). The new method takes into account and position of sample points on the interpolated point due to a coefficient $(\mathrm{K})$. The coefficient is added to set the sample point's distance weight due to its shielded effect in sample point positions. The proposed method (AIDW) has less mean related error $(6.5 \%)$ than the IDW method (7.1\%). Fan et al. [11] illustrate the evaluation effects of a uniform grid cell's size on the efficiency of k-nearest neighbor $(\mathrm{kNN})$ search to locate neighbors fast. The standard deviation of points' coordinates was used to measure the spatial distribution of scattered points. The relationships between the grid cell's optimal size and the standard deviation of scattered points' coordinates were fitted. This paper shows that with the increase of the standard deviation of points' coordinates, the grid cell's relatively optimal size decreases and converges.

\section{SPATIAL DETECTORS}

The surface EMG signal is the spatial and temporal summation of all active motor units' action potential under regional detection vacancy. A high-spatial-precision EMG electrode mounted on the skin surface of skeletal muscle under contraction allows detecting and estimating the size of a single motor unit (MU), in addition to analyzes of neurological properties like conduct velocity of a single motor unit action potential. It is based on the multi-electrode; it consists of multiple electrodes arranged in a set of one or two dimensions, with a distant space between the electrodes of 4 millimeters [12]-[16]. Figure 1 shows a multi-electrode grid, designed, and developed in the electronic circuits laboratoryldepartment of Electrical Engineering, University of Technology, Baghdad. It consists of $(4 \times 4)$ stainless steel pin-electrodes build on a plastic base and installed on a rubber layer to protect the skin of the subjects from damage. Because the signal's amplitude may vary from less than $50 \mu \mathrm{V}$ up to $30 \mathrm{mV}$, each picked signal is passed through a preamplifier, and the whole package is covered then by aluminum grounded shields to protect them from the noise. That was the detection, pre-amplification, and transduction stage of the signals.

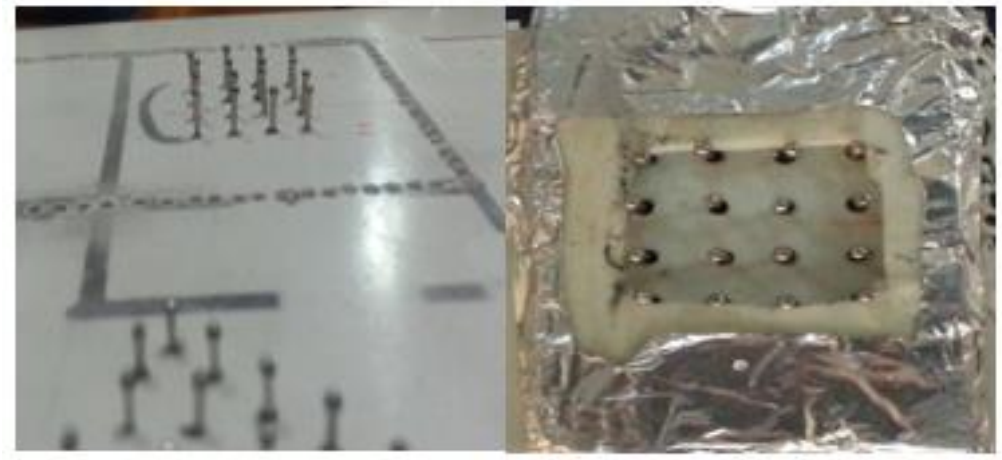

Figure 1. Electrode array with $(4 \times 4)$ electrodes

An electrode-amplifier circuit that detects the sEMG signal amplifies and eliminates some of the unwanted noise. The electrode amplifier has a high input impedance to restrict current drawn from the subject and reduce signal leakage and attenuation. Without a change in output voltage, the low output impedance drives the following electronic circuit stage. The input impedance of modern operational amplifiers is over $100 \mathrm{M} \Omega$, and the output impedance is $100 \Omega$. AD620 instrumentation amplifiers improve electronic efficiency (increase common-mode rejection ratio, decrease noise) and minimize inherent noise in subsequent stages. The amplifier is located very close to the electrodes, providing the most reliable sEMG information before amplification and reducing cable movement noise. Many researchers prefer this design because buffering the acquired signal near the source, amplifying the signal early in the process, and 
minimizing the physical measurements of a system by only including a pre-amplification stage near the subject attenuates noise and motion artifacts. For avoiding the intrusion of hazardous current into the subject, all components' power supply on the subject's side of the circuitry is isolated from the ground.

The second stage is to pass the 16-transduced analog signals to a 16-channel analog multiplexer/demultiplexer (CD74HC4067), multiplexer (CD74HC4067), which uses four digital pins to control the flow of one pin to 16 others. Next stage, the selected signal will be denoised by a band-pass filter $(15-500 \mathrm{~Hz})$ and then amplified. Finally, for the signal to be readable by the data acquisition system, a shifting level controlled by DC reference voltage $(2.5 \mathrm{~V})$ is applied.

Data acquisition system that is used for the proposed approach is based on the Arduino Uno platform. Arduino Uno microcontroller module has a USB connection interface. The Arduino IDE and libraries are open sources, and there are a large variety of accessories, shields that drop onto the microcontroller. It has a variety of I/O pins, including analog, digital, and postmenopausal women (PWM) ports. The block diagram of the system is shown in Figure 2.

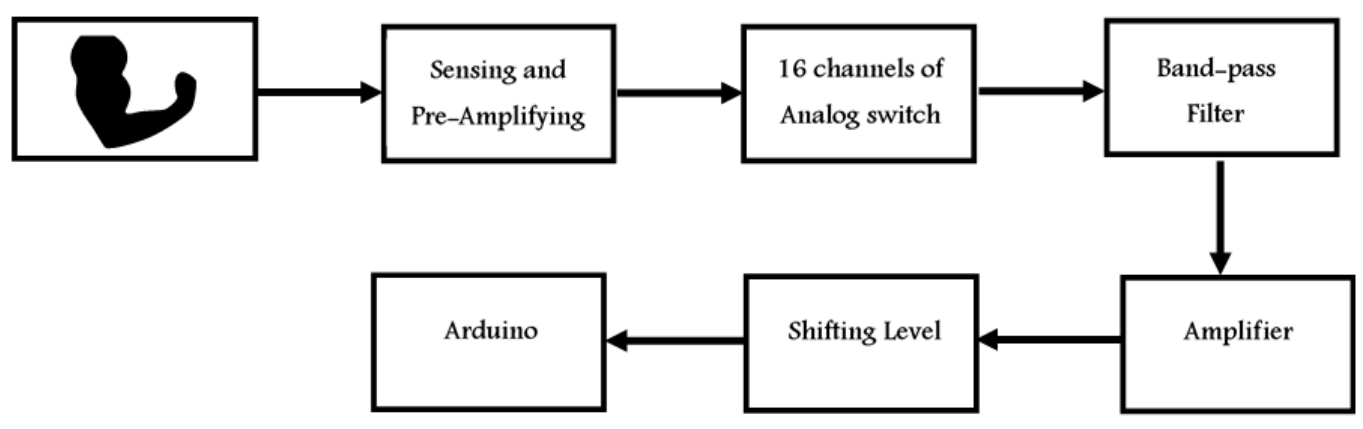

Figure 2. The block diagram of the system

When the skeletal muscle undergoes a voluntary contraction through nerve fiber, a possible time distribution variable of the potentials is detected on the skin's surface. Multi-electrode grids are used to observe the distribution of spatial potentials generated. From this, both localization of the potential source and its propagation in time can be obtained. The potentials detected by the grid's various electrodes can be considered the instantaneous spatial samples of all active MUs generated under the detection's vacancy by activating the muscle fibers. This potential distribution contributes in various ways, such that the MUs located near the surface of the skin contribute to the potential output distribution higher potential [13].

\section{SPATIAL INTERPOLATION}

Many interpolation techniques are available to predict the unknown area's values like spline, Kriging, trend, natural neighbors, and inverse distance weighting. The biggest question among the researchers is to find out which is the desirable method for interpolating data. Several discussions and researches have been conducted to find out the best interpolation by Mahalingam et al. [17]. Here, we present the idea of a variable signal over time, which represents the instant spatial samples of the possible dispersion of the muscle fibers. Two widely and robust techniques are chosen to compare with our proposed technique.

\subsection{Inverse distance weighted}

Spatial interpolation techniques are used to determine the unknown places using known location values. This paper presents a modified nonlinear IDW interpolation technique to estimate a missing EMG signal from available neighbor's signals. Normality and stationary assumptions are supposed to constrain the interpolation process within the data range. IDW is a robust and widely used technique to predict Olena's unknown point [18]. It is based on the presumption that the interpolating area should be affected essentially by the proximal points and minimal of the far away points. The interpolating point is a weighted rate of the nearby points, the weight assigned to each nearby point decreases as the distance from the interpolation point to the neighbor points increases. It is calculated as (1) [18].

$$
F(\boldsymbol{u})=\sum_{i=1}^{n} w_{i} f\left(\boldsymbol{u}_{i}\right)
$$


Where, $f(u)$ is the inverse distance estimation at location $\mathrm{u}, \mathrm{n}$ is the number of locations, $w_{i}, \mathrm{i}=1, \ldots ., \mathrm{n}$, are the weight attributed to each sample location, and $f\left(u_{i}\right)$ are the instantaneous data at the location $i$. The weights $w_{i}$ are determined by (2):

$$
w_{i}=\frac{\left(\frac{1}{d_{i}^{p}}\right)}{\sum_{i=1}^{n}\left(\frac{1}{d_{i}^{p}}\right)},(i, \ldots, n)
$$

where, $\left(\mathrm{w}_{\mathrm{i}}\right)$ are the euclidian distance between the interpolated data's location and used sample points, $\mathrm{p}$ is the distance value's power. Observe that:

$$
\sum_{i=1}^{n} w_{i}=1
$$

\subsection{Kriging}

Like IDW, Kriging uses a weighting, which assigns more influence on the nearest data points in the interpolation of values for unknown locations. However, Kriging is not deterministic but extends the proximity weighting approach of IDW to include random components where the function does not know its exact location [19]. Kriging depends on spatial and statistical relationships to calculate the surface. The two-step process of Kriging begins with semi variance estimations [20]. i) variance $=\left(\mathrm{Z}_{\mathrm{i}}-\mathrm{Z}_{\mathrm{j}}\right)^{2}$, ii) semi variance $=$ variance $/ 2$, iii) the interpolation by using semivariogram.

The advantages of this method are the incorporation of variable interdependence and the available error surface output. A disadvantage is that it requires substantially more computing and modeling time, and Kriging requires more input from the user (values and weights from every point) [21]. Kriging belongs to the family of linear least squares estimation algorithms. Kriging aims to estimate the value of an unknown real-valued function, $E$, at a point, $(x ; y)$, given the values of the function at some other points, $f(x 1 ; y 1) ;(x 2 ; y 2) ;(x 3 ; y 3) g$. A Kriging estimator is said to be linear because the predicted value $E(x ; y)$ is a linear combination that for each pair of points separated by distance (h): i) take the difference between the attribute values, ii) square it, iii) add to sum, and iv) divide the result by the number of pairs. As written:

$$
\gamma(h):=\frac{1}{|N(h)|} \sum_{(i, j) \in N(h)}\left|z_{i}-z_{j}\right|^{2}
$$

\section{SYSTEM SET-UP}

Four healthy subjects, males, and females (age 24-55 years) have different types of muscles, forearm flexor, extensor, Biceps Brachii, and m. Abductor Pollicisbrevis muscles are investigated. The study area consisted of a $3 \times 3$ dimensional arrangement of dry surface electrodes with a distant space of $5 \mathrm{~mm}$. Each electrode (1-9) will measure the muscle activity (monopolar potentials), EMG1, to EMG9 under the area of sensing concerning ground, as shown in Figure 3. The detected nine signals are amplified and fed into a computer via an A/D converter. For each test, a period of 5-second signals is sampled at a $4 \mathrm{kHz}$ rate and stored in the computer. A processing period of $100 \mathrm{msec}$ is considered appropriate for EMG signal processing and applications, as mentioned by Harba and Ibrahim [22]. Figure 4 shows the recorded nine real signals for the subject two configurations of the spatial electrodes used.

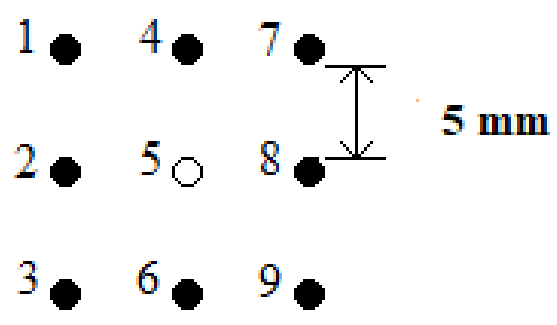

Figure 3. The arrangement of the electrodes 

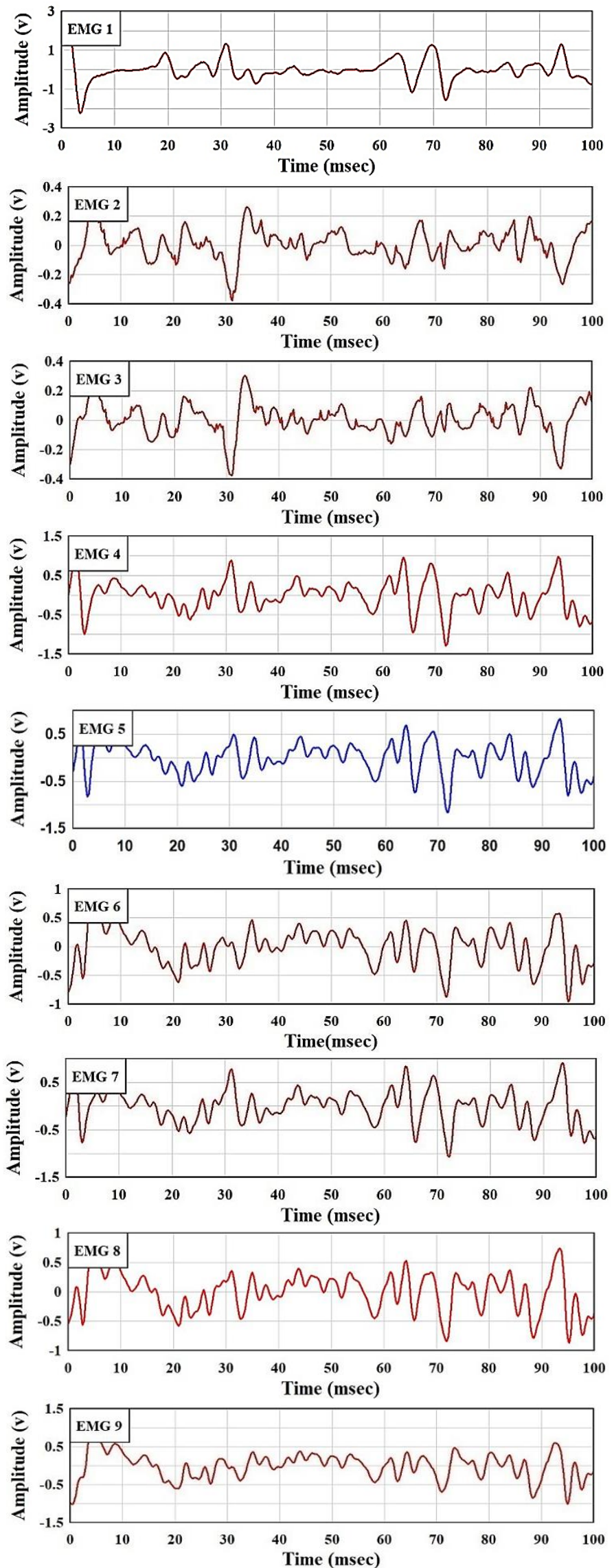

Figure 4. Recorded nine real signals for subject 2 


\section{IDW AND KRIGING TECHNIQUES}

The study area of interest is to use the IDW and Kriging interpolation techniques to interpolate the instantaneous value of the signal (EMG5) in the area surrounding by electrode locations $(1,7,3,9)$, from the instantaneous observation of the eight-neighbor signal's (EMG1, EMG2, EMG3, EMG4, EMG6, EMG7, EMG8, and EMG9). A goodness of fit (R2) is applied to measure the discrepancy between the interpolated and the real values of the signal (EMG 5),

$$
0 \geq R^{2} \geq 1
$$

where, 0: miss fitting, 1: good fitting.

Using the IDW technique, the power (p) of the Euclidian distance wi shown in (2), was investigated. Eight different power values $(0.5,1,2,5,10,15,25$, and 30) are selected to find the suitable value of the instantaneous interpolated signal (EMG5). A standard exponential model Kriging method is chosen to interpolate the missing EMG signal at point 5 from points (1, 2, 3, 6,9,8,7, and 4) as shown in Figure 1, which needs to estimate the nugget, sill, and the range used to describe the semivariance for each set of signals and subjects. Table 1 shows the Goodness of fit for both methods, offering the best power of distance for IDW. Results show that a better fit For the IDW method could be achieved as the power increased and settled in the order of $(\mathrm{p}=15)$ for subject $1(\mathrm{p}=12)$ for subject $2(\mathrm{p}=8)$ for subject three, and $(\mathrm{p}=5)$ for subject 10. In comparison, the Kriging method shows a slight improvement than the other. Figure 5 shows the real and interpolated EMG signals with a total processing delay time of $100 \mathrm{msec}$., When the muscles are under moderate contraction, for IDW $(\mathrm{p}=5)$ and Kriging interpolation method.

Table 1. IDW Interpolation for different module power

\begin{tabular}{ccccc}
\hline Interpolation methods & \multicolumn{4}{c}{ The Goodness of fit $\left(\mathrm{R}^{2}\right)$} \\
& Sub 1 & Sub 2 & Sub 7 & Sub 10 \\
\hline IDW & $0.8920(\mathrm{p}=15)$ & $0.7835(\mathrm{p}=12)$ & $0.8387(\mathrm{p}=8)$ & $0.7808(\mathrm{p}=5)$ \\
Kriging & 0.9215 & 0.80137 & 0.8543 & 0.8001 \\
\hline
\end{tabular}
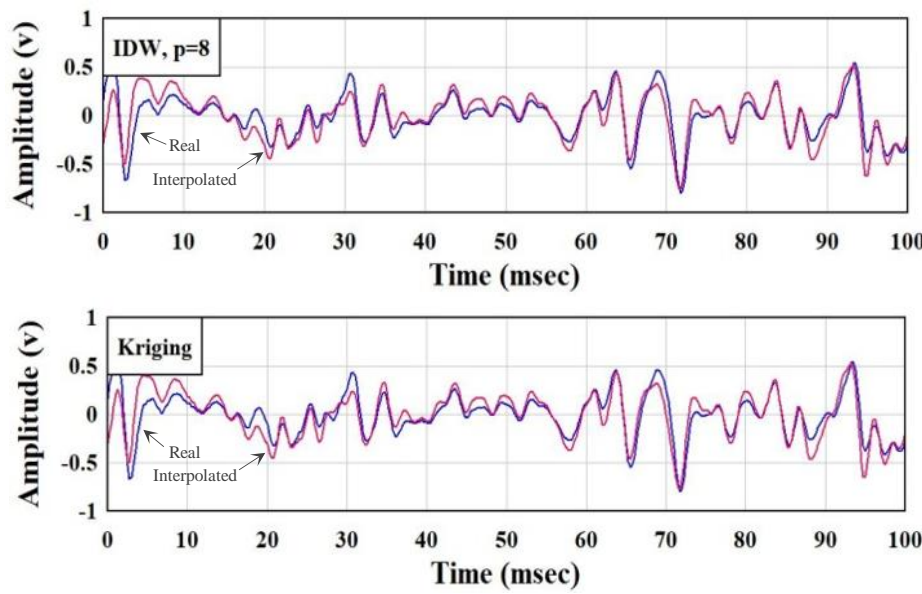

Figure 5. The real and interpolated signals using IDW and Kriging methods for subject 7

\section{THE PROPOSED TECHNIQUES}

Spatial data interpolation works based on the first law of geography formulated by Waldo Tobler [23]. The law provides that "all the features on the earth's surface have a relationship between one another, but the near features are having more relation than features away." However, for sEMG, that assumption is either valid if the detection area belongs to a single motor unit's activity or false if the detection area belongs to more than one motor unit. This assumption depends on the motor unit's size, which differs from muscle to muscle, as mentioned by Nandedkar et al. [24], and the detector's surface area. For this reason, further measures have been taken to study the effect of the presence of more than one motor unit under the area of detection. After evaluating the previous results, the number of recorded signals surrounding point 5 may have a weak effect on interpolating the missed signal, as shown in Figure 4. For that reason, two opposite spatial interpolation locations are implemented using IDW; Table 2 shows the effect of two opposite points to interpolate the signal at the location (5). The Goodness of fit is used to evaluate the interpolated signals 
concerning the real one. Results show that a better fit is achieved for locations 4 and 6 for all subjects, and that is true when the electrode axis is mounted parallel to the muscle's fibers, but this may not be valid if it is out of axis. In other cases, the fitness gets worse when the electrode axis is diverted toward the muscle fibers. There may be more than one motor unit under the area of detection. These results are seen to be correct as Figure 6 the amplitude of real and interpolated with order $(p=8)$ for the sEMG signals' cases, Figure 6(a) locations 1 and 9, Figure 6(b) locations 2 and 8, Figure 6(c) locations 3 and 7 and finally Figure 6(d) for locations 4 and 6 , with a total processing period of $100 \mathrm{msec}$, under a moderate muscle contraction. The best fit is obtained for interpolating the instantaneous signal at location 5 from instantaneous signals at locations 4 and 6 , and the worst fit is obtained at locations 3 and 7, while the other gives a moderate fit. That explains that there is more than one source of activity under the vacancy of detection, which will affect the instantaneous value of the interpolated signal.

From (1), the distance is only the criteria used to interpolate the signal, which satisfies the basis of the first law of geography formulated by Ouabo et al. [20]. In contrast, our results show that the detection area may be affected by more than one activity source for online interpolated signals. It was taken into consideration to develop a robust approach to interpolating the missed signal. For this basis, distance, and Goodness of fit (R2) between the real and interpolated signals were taken into consideration; a nonlinearity approach to the inverse distance is applied. A coefficient $(\mathrm{G})$, this nonlinearity is present, and the (1) is modified to:

$$
F(\boldsymbol{u})=K \cdot \sum_{i=1}^{n} w_{i} G_{i} f\left(\boldsymbol{u}_{i}\right)
$$

Gi has a coefficient derived from the normalized to one; the Goodness of fit is shown in Table 3.

A gain $(K)$ is made to overcome the interpolated signal's weakness by multiplying by the coefficients (Gi), normalized to one, to obtain the highest efficiency possible. The gain factor (K) is obtained by using the simple linear regression equation stated by [25], [26]:

$$
\hat{y}=a+b x
$$

The $y$-intercept is represented by (a), and the slope of the line is indicated by b. We obtain the simple linear regression equation using site sink source profiles (SSSP); by entering the $\mathrm{x}$ values as the quantized absolute mean value of each subject's eight surrounding signals. The output simple linear regression is (8):

$$
\hat{y}=4.985+2.685 x
$$

Which is more suitable for the best signal interpolated gain $(\mathrm{K})$ for all cases. Table 4 illustrates the Goodness of fit between the interpolated signals and real signal for the IDW and the modified IDW (proposed coefficient G). The result shows that the modified IDW spatial interpolation method has better-predicted accuracy than the standard IDW spatial interpolation, with an enhancement of $(9.85-26 \%)$.

The time responses, shown in Figure 7, show the power of matching between the spatial interpolation and the real sEMG signal, despite small distinction and the shape of the interpolated signal. The proposed technique shows reliability, confidentiality and can be applied to other types of biomedical signals. The non-normality shape of the signal distribution function and belongs to the non-convergence property is derived from the results of the conventional method in the case of non-contaminated distributions.

Table 2. IDW for different points

\begin{tabular}{cccccc}
\hline Subj. & Points & $4-6$ & $3-7$ & $2-8$ & $1-9$ \\
\hline 1 & $\mathrm{R}^{2}$ & 0.9671 & 0.7027 & 0.7603 & 0.7945 \\
2 & $\mathrm{R}^{2}$ & 0.9929 & 0.0650 & 0.7463 & 0.6998 \\
7 & $\mathrm{R}^{2}$ & 0.9756 & 0.6405 & 0.6019 & 0.6584 \\
10 & $\mathrm{R}^{2}$ & 0.9811 & 0.6450 & 0.7619 & 0.6230 \\
\hline
\end{tabular}

Table 3. The normalized Goodness of fit for different points $(\mathrm{G})$

\begin{tabular}{ccccc}
\hline Subj. & $4-6$ & $3-7$ & $2-8$ & $1-9$ \\
\hline 1 & 0.9671 & 0.7027 & 0.7603 & 0.7945 \\
2 & 0.9929 & 0.0650 & 0.7463 & 0.6998 \\
7 & 0.9756 & 0.6405 & 0.6019 & 0.6584 \\
10 & 0.9811 & 0.6450 & 0.7619 & 0.6230 \\
\hline
\end{tabular}

Table 4. The Goodness of fit between the real interpolated signals, before and after using the nonlinear factor $(\mathrm{G})$

\begin{tabular}{ccccc}
\hline Subj. & $\mathrm{R}^{2}$ (before) & $\mathrm{R}^{2}$ (after) & Improvement & $\mathrm{K}$ \\
\hline 1 & 0.8920 & 0.9803 & $9.8 \%$ & 5.5 \\
2 & 0.7835 & 0.9900 & $26.6 \%$ & 5.4 \\
7 & 0.8387 & 0.9620 & $14.7 \%$ & 5.7 \\
10 & 0.7808 & 0.9861 & $26.3 \%$ & 5.9 \\
\hline
\end{tabular}




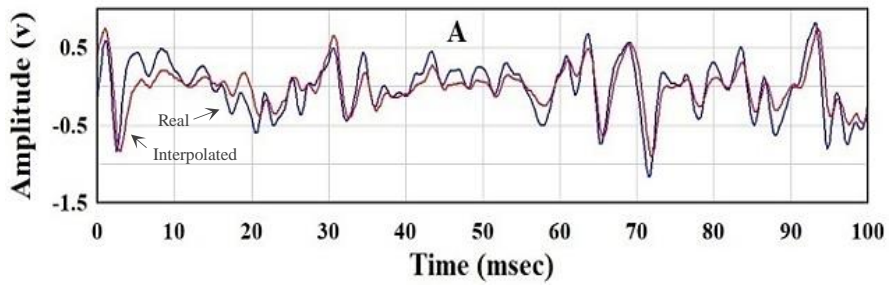

(a)

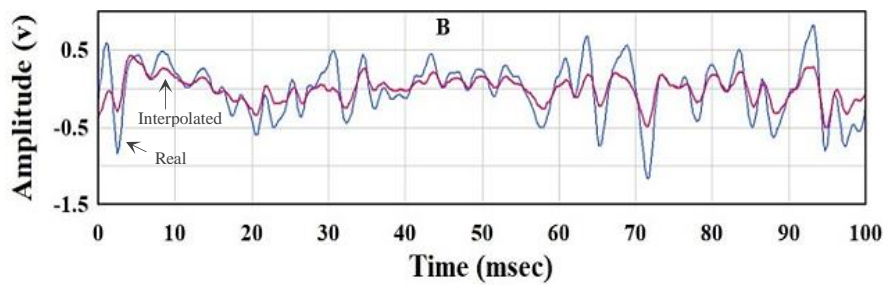

(b)

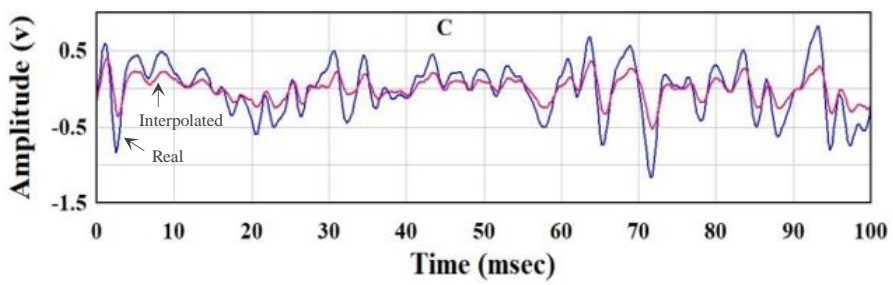

(c)

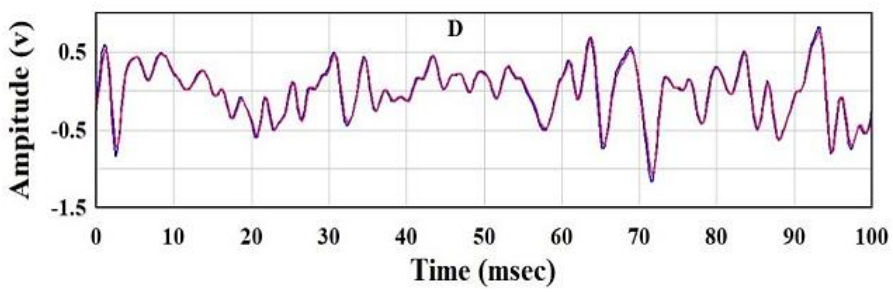

(d)

Figure 6. The real and interpolated signals for cases, (a) 1-9, (b) 2-8, (c) 3-7, and (d) 4-6

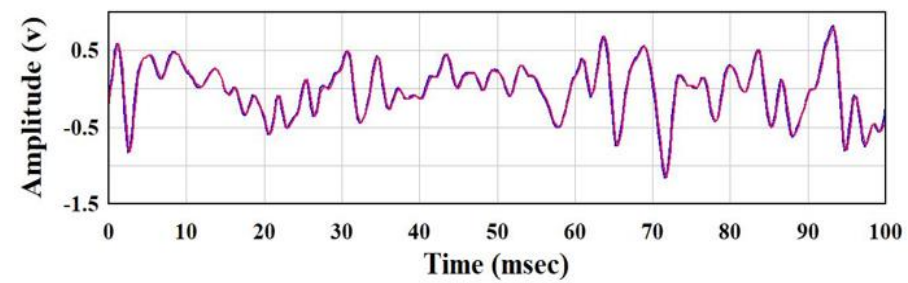

Figure 7. The real and spatial interpolated signals for subject 7

\section{CONCLUSION}

A new nonlinearities spatial interpolation formula is applied to interpolate the instantaneous value of the sEMG signal located at a specified location, by using the instantaneous observation of the surrounded neighbor's signals, to increase the electrodes' density in detecting the skeletal muscles' motor units under detection's vacancy. It must be mentioned that the results obtained are contrary to our hopes that the resultant technique is achieved better performance than the simple IDW. The time responses, shown in Figure 7, show the power of matching between the spatial interpolation and the real sEMG signal, despite the small distinction and the interpolated signal's shape. It can be concluded that the proposed technique shows reliability, confidentiality and can be applied to other types of biomedical signals. 
Hardware limitation of electrodes like technical problems and cost is an important issue that faces the EMG surface mounted electrode industry. The limited aperture between electrodes illustrates the technical problems. In contrast, the proposed system is a good solution for both issues (electrodes limitation and cost), by our modified technique to interpolate the desired signal extracted from known signals under the vacancy of the detection area. In other words, increase the precious detection of the electrodes. In the case of non-contaminated distributions, the suggested formula, which deals with the non-normality shape of the signal distribution function and belongs to the non-convergence property, derives from the results of the traditional method, so that the results are better than those of the conventional IDW method when dealing with contaminated distributions. The suggested formula has a high degree of accuracy between real and spatial interpolated signals regardless of different subjects or muscles, with an improvement in the Goodness of fit (9.85-26\%). One of the significant recommendations is to reconsider the characteristics of the missing signals, of the studied cases, through surrounded neighbor signals' instantaneous values. Furthermore, it is recommended that the suggested formula can be applied to the physical body's characteristics, such as the study of the motor unit action potential.

\section{REFERENCES}

[1] X. Hu, P. Yu, Q. Yu, W. Liu, and J. Qin, "Classification of surface EMG signal based on energy spectra change," in 2008 International Conference on BioMedical Engineering and Informatics, May 2008, pp. 375-379, doi: 10.1109/BMEI.2008.24.

[2] J. Chen, S. Bi, G. Zhang, and G. Cao, "High-density surface EMG-based gesture recognition using a 3D convolutional neural network," Sensors, vol. 20, no. 4, Art. no. 1201, Feb. 2020, doi: 10.3390/s20041201.

[3] D. Bai, S. Chen, and J. Yang, "Upper arm motion high-density sEMG recognition optimization based on spatial and timefrequency domain features," Journal of Healthcare Engineering, vol. 2019, pp. 1-16, Mar. 2019, doi: 10.1155/2019/3958029.

[4] "Interpolation methods|Human-robot interaction." 2014, Accessed: Oct. 10, $2020 . \quad$ [Online]. Available: https://hubotinteraction.wordpress.com/2014/03/28/interpolation-methods/.

[5] M. Pinheiro, P. Prats-Iraola, M. Rodriguez-Cassola, and M. Villano, "Analysis of low-oversampled staggered SAR data," IEEE Journal of Selected Topics in Applied Earth Observations and Remote Sensing, vol. 13, pp. 241-255, 2020, doi: 10.1109/JSTARS.2019.2959092.

[6] Y.-H. (Eva) Wu and M.-C. Hung, "Comparison of spatial interpolation techniques using visualization and quantitative assessment," in Applications of Spatial Statistics, InTech, 2016.

[7] N. Abser, D. MacIsaac, A. D. C. Chan, G. Fraser, and J. R. Green, "CLEANEMG: Comparing interpolation strategies for power line interference quantification in surface EMG signals," CMBEC, vol. 35, 2012.

[8] Y. Hu, Y. Li, and R. Song, "Robust interpolation of correspondences for large displacement optical flow," in 2017 IEEE Conference on Computer Vision and Pattern Recognition (CVPR), Jul. 2017, pp. 4791-4799, doi: 10.1109/CVPR.2017.509.

[9] I. J. R. Martinez, A. Mannini, F. Clemente, and C. Cipriani, "Online grasp force estimation from the transient EMG," IEEE Transactions on Neural Systems and Rehabilitation Engineering, vol. 28, no. 10, pp. 2333-2341, Oct. 2020, doi: 10.1109/TNSRE.2020.3022587.

[10] Z. Li, K. Wang, H. Ma, and Y. Wu, “An adjusted inverse distance weighted spatial interpolation method,” Proceedings of the 2018 3rd International Conference on Communications, Information Management and Network Security (CIMNS 2018), 2018, doi: 10.2991/cimns-18.2018.29.

[11] N. Fan, G. Mei, Z. Ding, S. Cuomo, and N. Xu, "Effects of spatial decomposition on the efficiency of k NN search in spatial interpolations," International Journal of Parallel, Emergent and Distributed Systems, pp. 1-19, Apr. 2020, doi: $10.1080 / 17445760.2020 .1758102$.

[12] C. Disselhorst-Klug, J. Silny, and G. Rau, "Estimation of the relationship between the noninvasively detected activity of single motor units and their characteristic pathological changes by modelling," Journal of Electromyography and Kinesiology, vol. 8, no. 5, pp. 323-335, Oct. 1998, doi: 10.1016/S1050-6411(98)00015-7.

[13] C. Disselhorst-Klug, J. Bahm, V. Ramaekers, A. Trachterna, and G. Rau, "Non-invasive approach of motor unit recording during muscle contractions in humans," European Journal of Applied Physiology, vol. 83, no. 2-3, pp. 144-150, Oct. 2000, doi: $10.1007 / \mathrm{s} 004210000272$.

[14] T.-Y. Sun, T.-S. Lin, and J.-J. Chen, "Multielectrode surface EMG for noninvasive estimation of motor unit size," Muscle and Nerve, vol. 22, no. 8, pp. 1063-1070, Aug. 1999, doi: 10.1002/(SICI)1097-4598(199908)22:8<1063::AID-MUS9>3.0.CO;2-R.

[15] D. Staudenmann, I. Kingma, D. F. Stegeman, and J. H. van Dieën, "Towards optimal multi-channel EMG electrode configurations in muscle force estimation: a high density EMG study," Journal of Electromyography and Kinesiology, vol. 15, no. 1, pp. 1-11, Feb. 2005, doi: 10.1016/j.jelekin.2004.06.008.

[16] I. Gligorijević, J. P. van Dijk, B. Mijović, S. Van Huffel, J. H. Blok, and M. De Vos, "A new and fast approach towards sEMG decomposition," Medical and Biological Engineering and Computing, vol. 51, no. 5, pp. 593-605, May 2013, doi: 10.1007/s11517-012-1029-y.

[17] B. Mahalingam, A. Deldar, and M. Vinay, "Analysis of selected spatial interpolation techniques for rainfall data," IJCRR, vol. 7, no. 7, 2015 .

[18] O. Babak and C. V Deutsch, "Statistical approach to inverse distance interpolation," Stochastic Environmental Research and Risk Assessment, vol. 23, no. 5, pp. 543-553, Jul. 2009, doi: 10.1007/s00477-008-0226-6.

[19] C. S. Pitombo, A. R. Salgueiro, A. S. G. da Costa, and C. A. Isler, "A two-step method for mode choice estimation with socioeconomic and spatial information," Spatial Statistics, vol. 11, pp. 45-64, Feb. 2015, doi: 10.1016/j.spasta.2014.12.002.

[20] R. E. Ouabo, A. Y. Sangodoyin, and M. B. Ogundiran, "Assessment of ordinary kriging and inverse distance weighting methods for modeling chromium and cadmium soil pollution in e-waste sites in Douala, Cameroon," Journal of Health and Pollution, vol. 10, no. 26, Art. no. 200605, Jun. 2020, doi: 10.5696/2156-9614-10.26.200605.

[21] M. A. Azpurua and K. Dos Ramos, "A comparison of spatial interpolation methods for estimation of average electromagnetic field magnitude," Progress In Electromagnetics Research M, vol. 14, pp. 135-145, 2010, doi: 10.2528/PIERM10083103.

[22] M. I. A. Harba and A. A. Ibraheem, "EMG processor based on the amplitude probability distribution," Journal of Biomedical 
Engineering, vol. 8, no. 2, pp. 105-114, Apr. 1986, doi: 10.1016/0141-5425(86)90044-0.

[23] W. R. Tobler, “A computer movie simulating urban growth in the detroit region," Economic Geography, vol. 46, Art. no. 234, Jun. 1970, doi: 10.2307/143141.

[24] S. D. Nandedkar, E. Stalberg, Y. I. Kim, D. B. Sanders, and A. Anne, "Use of signal representation to identify abnormal motor unit potentials in macro EMG," IEEE Transactions on Biomedical Engineering, vol. BME-31, no. 2, pp. 220-227, Feb. 1984, doi: 10.1109/TBME.1984.325332.

[25] W. Daniel and C. Cross, BIOSTATICS basic concepts and methodology for the health science. John Wiley \& Sons (Asia) Pte Ltd., 2010.

[26] K. J. Preacher, P. J. Curran, and D. J. Bauer, "Computational tools for probing interactions in multiple linear regression, multilevel modeling, and latent curve analysis," Journal of Educational and Behavioral Statistics, vol. 31, no. 4, pp. 437-448, Dec. 2006, doi: 10.3102/10769986031004437.

\section{BIOGRAPHIES OF AUTHORS}

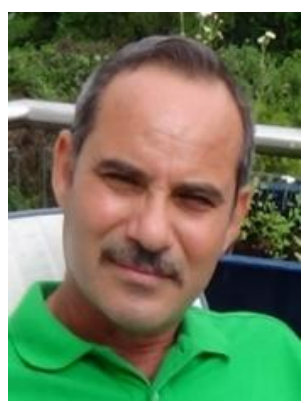

Ayad Assad Ibrahim (D) SC P was received his B.Sc., and M. Sc., degrees in 1982, and 1984 respectively from the University of Technology, Baghdad. He is currently a Lecturer in the Department of Electrical Engineering, University of Technology, Baghdad. His research interest is Biomedical Engineering and EMG signal processing. He has many publications in National and International Journals. Also, participate in many International Conferences in these mentioned fields. He can be contacted at email: 30055@uotechnology.edu.iq.

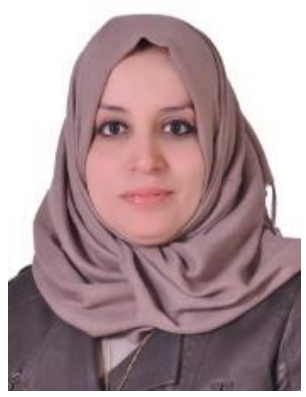

Ikhlas Mahmoud Farhan (iD SII SC P was received her B.Sc., and M. Sc., degrees in 2001, and 2013 respectively from the University of Technology, Baghdad. She is currently a Lecturer in the Department of Electrical Engineering, University of Technology, Baghdad. Her research interest is Electronic and communication Engineering, and DSP signal processing. She has many publications in National and International Journals. Also, participate in many International Conferences in these mentioned fields. He can be contacted at email: 30152@uotechnology.edu.iq.

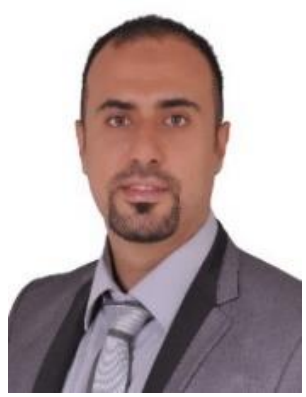

Mohammed Ehsan Safi (D) Pd SC P was received his B.Sc., and M. Sc., degrees in 2008, and 2013 respectively from the University of Technology, Baghdad. He is currently a Lecturer in the Department of Electrical Engineering, University of Technology, Baghdad. His research interest is Pattern Recognition, Image process, Biomedical: EMG signal processing, and smart system design. He has many publications in National and International Journals. Also, participate in many International Conferences in these mentioned fields. He can be contacted at email: 30165@uotechnology.edu.iq. 